\title{
Prevalence and recurrence of urinary tract and genital infections among adults with and without type 2 diabetes mellitus in the general population: a longitudinal cohort study
}

\author{
Susan Grandy ${ }^{1}$, Kathleen M. Fox ${ }^{2^{*}}$ and Elise Hardy ${ }^{1}$ \\ *Correspondence: kathyfox@gforcecable.com \\ ${ }^{1}$ AstraZeneca LP, Wilmington, DE, USA. \\ ${ }^{2}$ Strategic Healthcare Solutions, LLC, MD, USA.
}

\begin{abstract}
Background: This study estimated the prevalence, recurrence, and predisposing factors of self-reported urinary tract infections (UTIs) and genital infections among adults with and without type 2 diabetes mellitus (T2DM).

Methods: In the US Study to Help Improve Early evaluation and management of risk factors Leading to Diabetes (SHIELD) 2008 survey, respondents reported how many times in the past 12 months they had a UTI or genital infection. T2DM respondents were compared with respondents who did not have diabetes (No DM). T2DM respondents who reported 3 or more infections were classified as having recurrent infections and were compared with T2DM respondents who reported 1-2 infections (non-recurrent infections) and those without infections.

Results: The prevalence rate of UTI was $184.4 / 1000$ for T2DM and 127.8/1000 for No DM among women (p $<0.001$ ), and $50.9 / 1000$ for T2DM and 33.9/1000 for No DM among men $(p=0.02)$. Odds of experiencing a UTI were 1.5 times higher among T2DM respondents than No DM respondents. For T2DM respondents with UTI, 23\% reported recurrent UTIs, largely among women. Predictors of recurrent UTIs compared with no UTIs in women were overactive bladder, kidney problems, atherosclerosis, and COPD. Among men, the prevalence rate of balanitis was 41.8/1000 for T2DM and 19.2/1000 for No DM ( $\mathrm{p}<0.01$ ). Prevalence rate of vaginitis was $172.6 / 1000$ for T2DM and 125.0/1000 for No DM (p <0.01). Odds of vaginitis were 1.9 and balanitis were 2.3 times higher among T2DM than no DM respondents. Among T2DM women with vaginitis, 30\% reported recurrent vaginitis. Lower education, receiving oral anti-diabetic drugs plus insulin, and atherosclerosis predicted recurrent vaginitis compared with non-recurrent vaginitis.

Conclusions: UTIs and genital infections were more prevalent with T2DM than among respondents without diabetes. Recurrent infections occur in approximately $23 \%-30 \%$ of adults. Select characteristics may assist in identifying those likely to have self-reported recurrent infections.
\end{abstract}

Key words: Type 2 diabetes mellitus, urinary tract infections, genital infections, prevalence, recurrence

\section{Background}

Urinary tract infection (UTI) is the most common bacterial infection encountered in clinical practice [1]. UTIs accounted for almost 7 million office visits and 1 million emergency department visits according to the 1997 National Ambulatory Medical Care Survey and National Hospital Ambulatory Medical Care Survey [2]. Approximately half of all women have at least one symptomatic UTI during their lifetime $[3,4]$. Approximately $20 \%$ of all UTIs occur in men [5]. Relapse or reinfections are also a major concern. Many women experience relapses or reinfections of the lower urinary tract even after treatment with broad-spectrum antibiotics $[6,7]$.

Vaginitis is the most common gynecological condition encountered in clinical practice [8]. Among women with vaginitis, $40 \%-50 \%$ have at least one recurrence [8]. When recurrence is defined as 4 or more microbiologically documented episodes in a year, approximately $5 \%-7 \%$ of women will have recurrent Candida vaginitis [9].

Diabetes has been associated with an increased risk of UTI and genital infection [2]. Furthermore, patients with diabetes often have increased complications of UTI, including rare complications such as emphysematous cystitis, pyelonephritis, and fungal urinary tract infections (particularly Candida species), and infections are often more severe and associated with unusual manifestations [10]. Adults with diabetes are more susceptible to developing lower UTIs and genital infections due to various predisposing factors, such as hyperglycemia-related impairment of the immune response and glucosuria [10]. Anatomic and functional abnormalities of the urinary tract are also associated with diabetes, and these abnormalities complicate UTI [10]. A Dutch study showed that despite the fact that patients with diabetes more often received longer and more potent initial treatment than patients without diabetes, women with diabetes more often had 
recurrences of their UTIs [11]. However, there is limited information on the prevalence and recurrence of UTI and genital infection among diabetes patients in the United States. The present study estimated the prevalence and recurrence of self-reported UTI and genital infection among US adults with and without type 2 diabetes mellitus (T2DM). The present study also evaluated factors that might distinguish those with recurrent infections from those with non-recurrent or no infections among adults with T2DM.

\section{Methods}

A cross-sectional analysis of data collected in 2008 among respondents with and without T2DM was conducted using data from the Study to Help Improve Early evaluation and management of risk factors Leading to Diabetes (SHIELD) to ascertain prevalence, recurrence, and predisposing factors of UTIs and genital infections. SHIELD is a 5-year, survey-based study conducted to better understand patterns of health status, health behavior, and knowledge and attitudes of people living with diabetes and those with varying levels of cardiometabolic risk.

\section{SHIELD survey}

SHIELD included an initial screening phase to identify cases of interest in the general population (e.g., diabetes mellitus), and a baseline questionnaire to follow up identified cases and collect data regarding health status, health knowledge and attitudes, and current behaviors and treatments. Subsequently, annual follow-up surveys were administered for 5 years. A detailed description of the SHIELD methodology has been published previously $[12,13]$.

In brief, the screening survey was mailed in April 2004 to a stratified random sample of 200,000 US households, representative of the US population for geographic residence, household size and income, and age of head of household [14], identified by the Taylor Nelson Sofres National Family Opinion (TNS NFO) panel (Greenwich, CT). All TNS NFO surveys were voluntary, and no special incentives were provided. A response rate of $64 \%$ was obtained for the screening survey. The SHIELD study and 2008 survey were approved by the Quorum Review Board.

A comprehensive baseline survey was mailed in SeptemberOctober 2004 to a representative sample of individuals 18 years of age or older $(n=22,001)$, who were identified in the screening survey as having self-reported type 1 diabetes mellitus or T2DM, no diabetes (No DM), or being at risk for diabetes. Each respondent group was balanced to be representative of that segment of the population for age, gender, geographic region, household size, and income for the US population, and then a random sample from each group was selected and sent the baseline survey. A response rate of $72 \%$ was obtained for the baseline survey. The 2008 annual follow-up survey (response rate of 71\%) included the specific questions regarding the frequency of UTI and genital infection to assist in addressing a gap in knowledge regarding the impact of these infections among individuals with T2DM. Responses from the 2008 survey were analyzed and reported in this study.

\section{Study measures}

Respondents were classified as having T2DM based upon their self-report of having been told by a doctor, nurse, or other healthcare professional that they had T2DM. Individuals who did not report a diagnosis of T2DM, type 1 diabetes mellitus, or gestational diabetes were included as a comparison group (respondents with type 1 diabetes or gestational diabetes were excluded from the analysis). Respondents were asked "how many times in the past 12 months have you had an infection" (UTI separately from fungal/yeast infections of the genital area). Respondents with T2DM were classified as having recurrent infections (UTIs or genital infections) if they reported 3 or more infections in the past 12 months. Non-recurrent infections were defined as 1or 2 UTIs or genital infections as opposed to no infections. This definition of recurrence was selected based upon previous studies that used the criterion of 3 or more infections in a year [6,15-17], although there is no consensus or consistent definition for recurrence among other studies. Predisposing factors included demographic characteristics (age, race, and Hispanic ethnicity), household income (reported as $<\$ 30,000, \$ 30,000$ to $\$ 49,999, \$ 50,000$ to $\$ 74,999$, and $>\$ 75,000$ ), education (reported as high school degree or less, some college, college degree, or graduate studies), comorbid conditions, body mass index (BMI), and diabetes medications. Comorbid conditions were self-reported based on survey questions of being told by a healthcare professional that they had the condition. Overweight was defined as a BMI of $25.0-29.9 \mathrm{~kg} / \mathrm{m}^{2}$, and obese was defined as a BMI $>30 \mathrm{~kg} / \mathrm{m}^{2}$. Respondents reported all prescription medications that were currently prescribed for them at the time of the survey and were instructed to refer to their medication labels for accurate reporting. Diabetes medications were classified as oral antidiabetic medications (OADs) or insulin and respondents were classified as taking only OADs, only insulin, or a combination of OADs and insulin.

\section{Statistical analysis}

Respondents with T2DM were compared with respondents who did not report diabetes on the occurrence of UTIs and genital infections by gender. Comparisons between respondents with and without diabetes were conducted using chi-square test for categorical variables and t-tests for continuous variables. Logistic regression was used to adjust prevalence estimates for age and gender. For recurrence of infection, T2DM respondents reporting recurrent infections (UTIs or genital infections) were compared with T2DM respondents who reported non-recurrent infections and no infections. Comparisons between respondents with and without reported recurrent infections were 
Grandy et al. Journal of Diabetes Research \& Clinical Metabolism 2013,

http://www.hoajonline.com/journals/pdf/2050-0866-2-5.pdf

doi: 10.7243/2050-0866-2-5

Table 1. Characteristics of adult respondents with and without diabetes, $\mathrm{n}=\mathbf{1 1 , 5 7 8}$.

\begin{tabular}{lll}
\hline Characteristics & $\begin{array}{l}\text { T2DM } \\
(\mathbf{n}=\mathbf{2 , 6 7 1})\end{array}$ & $\begin{array}{l}\text { No diabetes } \\
(\mathbf{n}=\mathbf{8 , 9 0 7})\end{array}$ \\
\hline Age, years, mean (SD) & $63.1(11.7)^{*}$ & $56.9(15.5)$ \\
Women, \% & 60.3 & 62.3 \\
White, \% & $73.7^{*}$ & 67.0 \\
Education, high school degree or less, \% & $34.1^{*}$ & 28.2 \\
Household income <\$30,000, \% & $36.2^{*}$ & 27.9 \\
Overweight or obese, \% & $89.9^{*}$ & 75.6 \\
Number of comorbid conditions, mean & $5.3(3.1)^{*}$ & $3.3(2.7)$ \\
(SD) & & \\
Atherosclerosis, \% & $9.1^{*}$ & 4.7 \\
Cholesterol problem, \% & $74.5^{*}$ & 46.5 \\
Heart disease, \% & $24.0^{*}$ & 12.9 \\
Hypertension, \% & $72.4^{*}$ & 45.4 \\
\hline
\end{tabular}

${ }^{\star} \mathrm{p}<0.05 ; \mathrm{SD}=$ standard deviation; T2DM = type 2 diabetes mellitus.

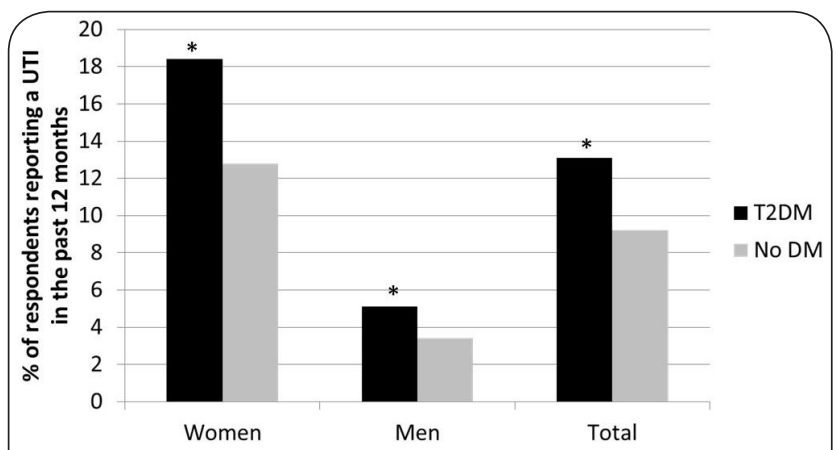

Figure 1. Respondents reporting at least 1 urinary tract infection by diabetes status and gender, $n=1,174 .{ }^{*} \mathrm{p}<0.01$ for comparison between T2DM and No DM; T2DM = type 2 diabetes mellitus; No DM = no diabetes mellitus.

conducted using chi-square test for categorical variables and t-tests for continuous variables. Logistic regression was used to determine predisposing factors for recurrent infections compared with non-recurrent infections and no infections. Odds ratio of having a recurrent infection with all baseline characteristics in the model were calculated. No adjustment was made for multiple comparisons and statistical significance was set a priori as $p<0.05$ to generate hypotheses.

\section{Results}

\section{Prevalence of urinary tract infections}

There were 2,671 respondents with T2DM and 8,907 respondents without diabetes who responded to the UTI survey questions (Table 1). A significantly greater proportion of T2DM respondents were older, white, had lower education and income, were overweight or obese, and had more comorbid conditions than respondents without diabetes (Table 1).

A total of $13.1 \%$ of respondents with T2DM and $9.2 \%$ of respondents without diabetes reported at least 1 UTI in the past 12 months ( $<<0.0001$; Figure 1$)$. Among women, $18.4 \%$ of those with T2DM, compared with $12.8 \%$ of those without diabetes, reported at least 1 UTI $(p<0.001)$. The prevalence rate of UTI for women was 184.4 per 1,000 people for those with T2DM, and 127.8 per 1,000 people for those without diabetes. Among men, $5.1 \%$ of those with T2DM, compared with $3.4 \%$ of those without diabetes, reported at least 1 UTI $(p=0.01)$. The prevalence rate of UTI for men was 50.9 per 1,000 people for those with T2DM and 33.9 per 1,000 people for those without diabetes. A higher proportion of women with T2DM than men with T2DM reported at least 1 UTI $(p<0.01)$.

The odds of having at least 1 UTI were 1.5 times higher among T2DM respondents than No DM respondents, after adjusting for age and gender (Table 2). Women were 4.2 times more likely than men to have at least 1 UTI, after adjusting for diabetes status and age. Respondents who were 80 years of age or older were more likely than younger ( $<50$ years of age) respondents to have a UTI, after adjusting for diabetes status and gender. Other age groups were not associated with increased odds of having a UTI compared with those $<50$ years of age.

\section{Recurrent urinary tract infections in T2DM}

Among the 2,671 respondents with T2DM who completed the UTI survey questions, 351 respondents reported having UTIs, and 2,320 reported no UTIs. Among the T2DM respondents reporting UTIs, 23.1\% reported having recurrent UTIs ( 3 or more in the past 12 months), and $76.9 \%$ reported having non-recurrent UTIs ( 1 or 2 in the past 12 months). Approximately $64 \%$ of T2DM respondents reporting recurrent UTIs had 3-4 infections and $36 \%$ had 5 or more in the past 12 months. The majority (73.7\%) of T2DM respondents reporting non-recurrent UTIs had only 1 infection in the past 12 months. Of the T2DM respondents with recurrent UTIs, 8 were men and 73 were women.

\section{Predisposing factors for recurrent urinary tract infections in T2DM}

T2DM respondents reporting recurrent UTIs were similar to T2DM respondents reporting non-recurrent UTIs, except for arthritis, where a larger proportion of the recurrent UTIs group (69\%) had arthritis than the non-recurrent UTI group (54\%, $p<0.05)$. T2DM respondents reporting recurrent UTIs did not differ from those reporting nonrecurrent UTIs on age, gender, race, education, income, $\mathrm{BMI}$, duration of diabetes, insulin use, or other comorbid conditions. Compared with T2DM respondents reporting no UTIs, a significantly greater proportion of T2DM respondents reporting recurrent UTIs were women $(90 \%$ vs. $57 \%$ ), had lower education ( $46 \%$ vs. $33 \%$ with high school degree or less), lower income ( $57 \%$ vs. $34 \%$ with $<\$ 30,000)$, higher BMI (37.0 [SD 9.8] vs. 33.8 [8.0]), longer diabetes duration (13.9 [10.5] years vs. 11.3 [8.5]), more prevalent use of insulin ( $29 \%$ vs. $20 \%$ ), and more comorbid 
Table 2. Odds ratio (and 95\% confidence intervals) of having a urinary tract infection or genital infection in the past 12 months, adjusting for age and gender.

\begin{tabular}{|c|c|c|c|c|c|c|}
\hline & $\begin{array}{l}\text { Urinary tract infectio } \\
N=11,578\end{array}$ & & Vaginitis $N=6,758$ & & Balanitis $\mathrm{N}=3,652$ & \\
\hline Characteristics & Odds ratio $(95 \% \mathrm{CI})$ & p-value & Odds ratio $(95 \% \mathrm{CI})$ & p-value & Odds ratio $(95 \% \mathrm{CI})$ & p-value \\
\hline $\begin{array}{l}\text { T2DM } \\
\text { (No DM as reference group) }\end{array}$ & $1.54(1.34-1.76)$ & $<0.0001$ & $1.90(1.62-2.24)$ & $<0.0001$ & $2.27(1.46-3.53)$ & $<0.0001$ \\
\hline $\begin{array}{l}\text { Age group } 50-59 \text { years (age } \\
\text { group }<50 \text { years as reference } \\
\text { group) }\end{array}$ & $0.95(0.80-1.23)$ & 0.54 & $0.57(0.48-0.68)$ & $<0.0001$ & $1.00(0.55-1.82)$ & 0.99 \\
\hline $60-69$ years & $0.90(0.75-1.08)$ & 0.27 & $0.31(0.25-0.38)$ & $<0.0001$ & $1.00(0.55-1.81)$ & 0.99 \\
\hline $70-79$ years & $1.08(0.90-1.32)$ & 0.40 & $0.24(0.18-0.32)$ & $<0.0001$ & $0.69(0.33-1.41)$ & 0.31 \\
\hline$\geq 80$ years & $1.52(1.21-1.90)$ & $<0.0001$ & $0.16(0.10-0.26)$ & $<0.0001$ & $0.98(0.43-2.24)$ & 0.96 \\
\hline $\begin{array}{l}\text { Women } \\
\text { (men as reference group) }\end{array}$ & $4.24(3.58-5.02)$ & $<0.0001$ & NA & - & NA & - \\
\hline
\end{tabular}

T2DM = type 2 diabetes mellitus; No DM = no diabetes mellitus; NA = not applicable.

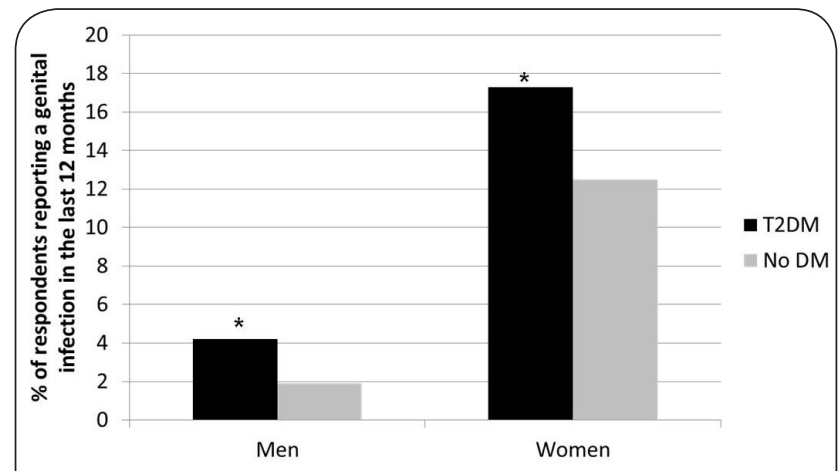

Figure 2. Men and women reporting at least 1 genital infection by diabetes status, $\mathrm{n}=1,008 .{ }^{*} \mathrm{p}<0.001$ for comparison between T2DM and No DM; T2DM = type 2 diabetes mellitus; No DM = no diabetes mellitus.

conditions ( $17 \%$ vs. $9 \%$ narrow/blocked arteries, $69 \%$ vs. $48 \%$ arthritis, $26 \%$ vs. $8 \%$ kidney problems, $36 \%$ vs. $10 \%$ overactive bladder/incontinence) ( $p<0.05$ for each).

Using logistic regression to control for the differences between groups, predisposing factors for recurrent UTIs were elucidated for women. There were too few men $(n=$ 8) with recurrent UTIs for the regression analysis. Among T2DM women, the odds of having recurrent UTIs compared with no UTIs were significantly higher if respondents had overactive bladder/ incontinence (OR: 3.6; 95\% Cl: 1.96.6), kidney problems (OR: $2.6 ; 95 \% \mathrm{Cl}: 1.2-5.4)$, narrow or blocked arteries (OR: 2.8; 1.0-7.3) (Table 3). T2DM women who reported having chronic obstructive pulmonary disease (COPD) had significantly lower odds of having recurrent UTIs compared with no UTIs $(p=0.035)$. Age, race, $\mathrm{BMI}$, duration of diabetes, household income, education, health insurance, insulin treatment, and other comorbid conditions did not significantly predict the likelihood of recurrent UTIs among the T2DM women. Compared with T2DM women who reported non-recurrent UTIs, the odds of having recurrent UTIs were significantly lower if respondents reported COPD or cardiac revascularization (e.g., angioplasty or bypass surgery) $(p<0.02)$ (Table 3$)$. Age, race, BMI, duration of diabetes, household income, education, health insurance, insulin treatment, and most comorbid conditions did not significantly predict the likelihood of recurrent UTIs compared with non-recurrent UTIs among the T2DM women.

\section{Prevalence of genital infections}

There were 2,421 respondents with T2DM (886 men, 1,535 women) and 7,989 respondents without diabetes (2,766 men, 5,223 women) who responded to the genital infection survey questions. The characteristics of these respondents were similar to those reported in Table 1.

For men, $4.2 \%(n=37)$ of respondents with T2DM and $1.9 \%(n=53)$ of respondents without diabetes reported at least 1 genital infection (i.e., balanitis) in the past 12 months (Figure 2). Among men, the prevalence rate of balanitis was 41.8 per 1,000 people for those with T2DM, and 19.2 per 1,000 people for those without diabetes $(p=$ $0.0003)$. For women, $17.3 \%(n=265)$ of respondents with T2DM and $12.5 \%(n=653)$ of respondents without diabetes reported at least 1 genital infection (i.e., vaginitis) in the past 12 months (Figure 2). The prevalence rate of vaginitis was 172.6 per 1,000 people for those with T2DM, and 125.0 per 1,000 people for those without diabetes $(p<0.0001)$.

The odds of having at least 1 vaginitis infection were 1.90 times $(95 \% \mathrm{Cl}=1.62-2.24)$ higher among T2DM than No DM women, after adjusting for age (Table 2). The odds of having at least 1 balanitis infection was 2.27 times (1.46-3.53) higher among T2DM than No DM men, after adjusting for age. The odds of having a vaginitis infection steadily declined with older age after adjusting for diabetes status: $\mathrm{OR}=0.57$ (95\% Cl: 0.48-0.68) for age group 50-59 years and $\mathrm{OR}=0.24$ (95\% Cl: $0.18-0.32)$ for age group 70-79 years compared with age group $<50$ years $(p<0.0001$ 
Grandy et al. Journal of Diabetes Research \& Clinical Metabolism 2013,

http://www.hoajonline.com/journals/pdf/2050-0866-2-5.pdf

doi: 10.7243/2050-0866-2-5

Table 3. Odds ratio (and 95\% confidence intervals) of having recurrent urinary tract infections (UTIs) or recurrent vaginitis in the past 12 months compared with no infections or non-recurrent infection for women.

\begin{tabular}{|c|c|c|c|c|c|c|c|c|}
\hline \multirow[b]{2}{*}{ Characteristics } & \multicolumn{2}{|c|}{$\begin{array}{l}\text { Recurrent UTIs vs. No UTIs } \\
\mathrm{N}=\mathbf{1 , 2 5 0}\end{array}$} & \multicolumn{2}{|c|}{$\begin{array}{l}\text { Recurrent UTIs vs. } \\
\text { Non-recurrent UTIs N= 271 }\end{array}$} & \multicolumn{2}{|l|}{$\begin{array}{l}\text { Recurrent Vaginitis vs. } \\
\text { No Vaginitis } N=1,223\end{array}$} & \multicolumn{2}{|c|}{$\begin{array}{l}\text { Recurrent Vaginitis vs. } \\
\text { Non-recurrent Vaginitis } \mathrm{N}=\mathbf{2 4 4}\end{array}$} \\
\hline & Odds ratio $(95 \% \mathrm{CI})$ & p-value & Odds ratio (95\% CI) & p-value & Odds ratio (95\% CI) & p-value & Odds ratio $(95 \% \mathrm{CI})$ & p-value \\
\hline Age, years (continuous) & $0.99(0.97-1.02)$ & 0.69 & $1.02(0.98-1.05)$ & 0.33 & $0.93(0.90-0.95)$ & $<0.0001$ & $0.99(0.96-1.02)$ & 0.67 \\
\hline $\begin{array}{l}\text { White (reference: other } \\
\text { races than black) }\end{array}$ & $0.60(0.28-1.29)$ & 0.19 & $0.76(0.30-1.90)$ & 0.56 & $1.21(0.64-2.31)$ & 0.56 & $1.49(0.67-3.32)$ & 0.33 \\
\hline $\begin{array}{l}\text { Black (reference: other } \\
\text { minority races) }\end{array}$ & $0.57(0.15-2.21)$ & 0.42 & $0.11(0.01-1.15)$ & 0.06 & $0.61(0.16-2.23)$ & 0.45 & $0.78(0.14-4.32)$ & 0.77 \\
\hline Hispanic (reference: no) & $0.88(0.25-3.13)$ & 0.85 & $0.78(0.19-3.25)$ & 0.73 & $0.41(0.09-1.85)$ & 0.24 & $0.74(0.13-4.19)$ & 0.73 \\
\hline $\begin{array}{l}\text { Body mass index, } \mathrm{kg} / \mathrm{m}^{2} \\
\text { (continuous) }\end{array}$ & $1.01(0.98-1.05)$ & 0.45 & $1.01(0.98-1.05)$ & 0.47 & $0.99(0.96-1.02)$ & 0.45 & $1.04(1.00-1.08)$ & 0.07 \\
\hline $\begin{array}{l}\text { Duration of diabetes, } \\
\text { years (continuous) }\end{array}$ & $1.02(0.98-1.05)$ & 0.37 & $0.99(0.96-1.03)$ & 0.66 & $1.00(0.97-1.04)$ & 0.91 & $0.97(0.93-1.02)$ & 0.25 \\
\hline $\begin{array}{l}\text { Household income, } \\
\text { (reference: } \geq \$ 75,000 \text { ) }\end{array}$ & - & - & - & - & - & - & - & - \\
\hline$<\$ 30,000$ & $1.57(0.63-3.91)$ & 0.33 & $1.18(0.41-3.42)$ & 0.76 & $1.61(0.64-4.03)$ & 0.31 & $0.82(0.28-2.43)$ & 0.72 \\
\hline$\$ 30,000-\$ 49,999$ & $1.05(0.39-2.81)$ & 0.93 & $0.92(0.29-2.98)$ & 0.89 & $1.22(0.47-3.14)$ & 0.68 & $1.01(0.31-3.29)$ & 0.99 \\
\hline$\$ 50,000-\$ 74,999$ & $1.10(0.38-3.13)$ & 0.86 & $1.32(0.36-4.83)$ & 0.67 & $1.07(0.38-3.03)$ & 0.90 & $1.06(0.30-3.69)$ & 0.93 \\
\hline $\begin{array}{l}\text { Education (reference: } \\
\text { some college or higher) }\end{array}$ & - & - & - & - & - & - & - & - \\
\hline $\begin{array}{l}\text { High school degree } \\
\text { or less }\end{array}$ & $1.36(0.78-2.40)$ & 0.28 & $1.07(0.54-2.12)$ & 0.85 & $1.45(0.84-2.48)$ & 0.18 & $1.98(1.02-3.84)$ & 0.043 \\
\hline $\begin{array}{l}\text { Health insurance (refer- } \\
\text { ence: no insurance) }\end{array}$ & $0.98(0.38-2.58)$ & 0.98 & $0.76(0.24-2.44)$ & 0.65 & $0.50(0.25-1.00)$ & 0.051 & $0.51(0.21-1.24)$ & 0.14 \\
\hline $\begin{array}{l}\text { Diabetes treatment (ref- } \\
\text { erence: no treatment) }\end{array}$ & - & - & - & - & - & - & - & - \\
\hline OADs alone & $1.14(0.45-2.85)$ & 0.79 & $1.65(0.56-4.86)$ & 0.36 & $2.29(0.84-6.25)$ & 0.10 & $2.95(0.92-9.47)$ & 0.07 \\
\hline Insulin alone & $1.70(0.51-5.65)$ & 0.39 & $2.00(0.50-8.04)$ & 0.33 & $2.22(0.62-7.89)$ & 0.22 & $2.04(0.44-9.48)$ & 0.36 \\
\hline OAD + insulin & $1.68(0.56-5.03)$ & 0.35 & $3.80(0.98-14.83)$ & 0.054 & $6.07(2.04-18.06)$ & 0.001 & $4.31(1.19-15.57)$ & 0.026 \\
\hline $\begin{array}{l}\text { Comorbid conditions } \\
\text { (reference for each: } \\
\text { absence of condition) }\end{array}$ & - & - & - & - & - & - & - & - \\
\hline $\begin{array}{l}\text { Overactive bladder/ } \\
\text { incontinence }\end{array}$ & $3.60(1.95-6.64)$ & $<0.0001$ & $2.07(0.99-4.34)$ & 0.053 & $3.29(1.69-6.41)$ & $<0.0001$ & $1.22(0.52-2.86)$ & 0.66 \\
\hline Kidney problems & $2.56(1.23-5.35)$ & 0.012 & $1.51(0.62-3.69)$ & 0.36 & $2.00(0.91-4.40)$ & 0.08 & $1.00(0.37-2.73)$ & 1.00 \\
\hline $\begin{array}{l}\text { Chronic kidney disease/ } \\
\text { end-stage renal disease }\end{array}$ & $0.91(0.23-3.58)$ & 0.89 & $0.70(0.15-3.29)$ & 0.65 & $1.36(0.45-4.13)$ & 0.58 & $3.11(0.70-13.78)$ & 0.14 \\
\hline $\begin{array}{l}\text { Narrow or blocked } \\
\text { arteries }\end{array}$ & $2.76(1.04-7.27)$ & 0.041 & $2.67(0.78-9.10)$ & 0.12 & $4.46(1.70-11.71)$ & 0.002 & $3.83(1.05-13.95)$ & 0.042 \\
\hline Hypertension & $1.33(0.65-2.76)$ & 0.44 & $1.32(0.56-3.09)$ & 0.53 & $0.97(0.52-1.84)$ & 0.94 & $0.83(0.38-1.84)$ & 0.65 \\
\hline Heart disease & $0.78(0.35-1.75)$ & 0.55 & $1.16(0.44-3.03)$ & 0.76 & $0.44(0.18-1.08)$ & 0.07 & $0.67(0.20-2.23)$ & 0.52 \\
\hline Cholesterol problems & $1.16(0.60-2.24)$ & 0.66 & $1.78(0.81-3.94)$ & 0.15 & $1.08(0.58-2.03)$ & 0.80 & $1.03(0.48-2.20)$ & 0.94 \\
\hline $\begin{array}{l}\text { Stroke or transient } \\
\text { ischemic attack }\end{array}$ & $0.47(0.13-1.76)$ & 0.26 & $0.33(0.07-1.58)$ & 0.17 & $0.99(0.38-2.56)$ & 0.99 & $6.38(0.99-41.06)$ & 0.051 \\
\hline $\begin{array}{l}\text { Angioplasty, stent, or } \\
\text { bypass surgery }\end{array}$ & $0.40(0.09-1.71)$ & 0.22 & $0.14(0.03-0.77)$ & 0.023 & $0.41(0.11-1.56)$ & 0.19 & $1.17(0.19-7.11)$ & 0.86 \\
\hline Heart attack & $0.48(0.09-2.66)$ & 0.40 & $0.24(0.04-1.46)$ & 0.12 & $3.10(0.92-10.46)$ & 0.07 & $1.05(0.21-5.13)$ & 0.96 \\
\hline Circulation problems & $1.49(0.78-2.85)$ & 0.23 & $0.94(0.43-2.06)$ & 0.88 & $2.23(1.21-4.09)$ & 0.010 & $1.40(0.64-3.04)$ & 0.40 \\
\hline $\begin{array}{l}\text { Chronic obstructive } \\
\text { pulmonary disease }\end{array}$ & $0.11(0.02-0.86)$ & 0.035 & $0.04(0.003-0.38)$ & 0.006 & $0.77(0.30-1.96)$ & 0.59 & $1.29(0.40-4.12)$ & 0.67 \\
\hline Arthritis & $1.17(0.62-2.18)$ & 0.63 & $1.23(0.60-2.55)$ & 0.57 & $1.01(0.57-1.80)$ & 0.96 & $0.84(0.43-1.63)$ & 0.60 \\
\hline Cancer, except skin & $1.24(0.60-2.58)$ & 0.56 & $0.80(0.34-1.87)$ & 0.61 & $1.48(0.71-3.09)$ & 0.29 & $1.63(0.67-3.99)$ & 0.28 \\
\hline
\end{tabular}

$\mathrm{OAD}=$ oral anti-diabetic agent.

for both). Age was not a significant predictor of balanitis.

\section{Recurrent genital infections in T2DM}

Among 1,535 women with T2DM, 80 (5.4\%) reported recurrent vaginitis (at least 3 infections in the past 12 months), 185 (12.6\%) reported non-recurrent vaginitis, and 1,270 (82.0\%) reported no genital infections. Among T2DM women with vaginitis $(n=265), 30.2 \%$ reported recurrent vaginitis. Approximately $61 \%$ of T2DM women with recurrent vaginitis reported 3-4 infections, and $39 \%$ had 5 or more vaginal infections in the past 12 months. The majority (65\%) of T2DM women reporting non-recurrent vaginitis had only 1 infection in the past 12 months. Among 886 men with T2DM, 10 (1.1\%) reported recurrent balanitis, 27 (3.0\%) reported non-recurrent balanitis, and 849 (95.8\%) reported no genital infections.

Predisposing factors for recurrent vaginitis in T2DM Because there were so few men with recurrent balanitis, 
the analysis of predisposing factors focused on recurrent vaginitis. Among T2DM respondents, women reporting recurrent vaginitis had significantly higher BMI $(38.4 \mathrm{~kg} /$ $\mathrm{m}^{2}$ [SD 10.1] vs. 35.7 [7.6], $\left.\mathrm{p}<0.05\right)$, greater proportion with narrow or blocked arteries ( $17.5 \%$ vs. $5.4 \%, \mathrm{p}<0.05)$, and circulation problems ( $35.0 \%$ vs. $23.2 \%, p<0.05)$, and lower proportion with health insurance $(76.3 \%$ vs. $89.7 \%$, $\mathrm{p}<0.05)$, compared with women with non-recurrent vaginitis. Compared with T2DM with no vaginitis in the past 12 months, T2DM women with recurrent vaginitis were younger (54.9 [SD 10.9] vs. 62.9 [11.5], $\mathrm{p}<0.05)$, had lower income ( $55.0 \%$ vs. $40.9 \%$ with income $<\$ 30,000$, p $<0.05)$, higher BMI (38.4 vs. 35.2, $\mathrm{p}<0.05)$, greater proportion with narrow or blocked arteries $(17.5 \%$ vs. $7.0 \%, p<0.05)$, circulation problems $(35.0 \%$ vs. $18.3 \%, \mathrm{p}<0.05)$, overactive bladder $(23.8 \%$ vs. $12.7 \%, \mathrm{p}<0.05)$, kidney problems $(18.8 \%$ vs. $7.5 \%, p<0.05)$, lower proportion with health insurance (76.3\% vs. $91.9 \%, \mathrm{p}<0.05)$, and more used insulin $(39.0 \%$ vs. $19.2 \%, p<0.05)$.

Using logistic regression with all characteristics included in the model, the odds of recurrent vaginitis compared with no vaginitis were 6.1 times higher if T2DM women received insulin plus oral anti-diabetic drugs compared with T2DM women who were not treated $(p=0.001)$ (Table 3). Other factors significantly increasing the odds of recurrent vaginitis among T2DM women were younger age ( $7 \%$ per year), overactive bladder (3.3 times higher odds), narrow or blocked arteries (4.5 times higher odds), and circulation problems $(\mathrm{OR}=2.2)$. Race, $\mathrm{BMI}$, duration of diabetes, household income, education, health insurance, and other comorbid conditions did not significantly predict the likelihood of recurrent vaginitis compared with no vaginitis among T2DM women. Compared with T2DM women who reported non-recurrent vaginitis, the odds of having recurrent vaginitis were significantly higher if women had less education (2 times higher), received insulin plus oral anti-diabetic drugs (4 times higher), or had narrow or blocked arteries (3.8 times higher) (Table 3). Age, race, BMI, duration of diabetes, household income, health insurance, and other comorbid conditions did not significantly predict the likelihood of recurrent vaginitis compared with non-recurrent vaginitis among T2DM women.

\section{Discussion}

UTIs were more prevalent among respondents with T2DM, compared with respondents without diabetes. UTIs were more frequent among women with T2DM, compared with men with T2DM and men without diabetes. Among T2DM respondents with at least 1 UTI reported, approximately $25 \%$ had recurrent UTIs ( 3 or more in the past 12 months); $25 \%$ for women and $15 \%$ for men. Specific demographic and clinical characteristics available in routine clinical practice predicted recurrent UTIs compared with nonrecurrent or no UTIs in women, including overactive bladder/incontinence, kidney problems, and narrow or blocked arteries. T2DM women with COPD have a lower risk of recurrent UTIs. These characteristics may assist in identifying those likely to have recurrent UTIs.

Vaginitis and balanitis are also more prevalent among individuals with T2DM compared with respondents without diabetes. Among women with T2DM reporting vaginitis, approximately $30 \%$ self-reported having recurrent vaginitis. Key demographics (age, education) and comorbid conditions (circulation problems, overactive bladder, narrow or blocked arteries) increased the odds of recurrent vaginitis versus non-recurrent vaginitis or no vaginitis. These characteristics available in routine clinical practice may assist patients and physicians to identify those likely to have recurrent vaginitis. The impact of these infections on healthcare resource utilization, including emergency room care, time lost from work, and overall healthcare costs for treating UTIs and genital infections is significant $[2,5]$.

The findings from the SHIELD study confirm previous reports that UTIs and genital infections are common $[8,18]$ and are more common among individuals with T2DM $[2,10,11]$. Moreover, the SHIELD findings are one of the first to provide prevalence estimates of UTIs, genital infections, and their recurrence among individuals with T2DM in the United States. SHIELD also confirmed earlier reports that UTIs were significantly more likely to occur among women than men [2] and that adults with T2DM more often had recurrences of UTIs than adults without diabetes [11]. SHIELD had a lower rate of UTIs in men compared with national estimates in 2000: 33.9 per 1,000 among those without diabetes vs. 136.9/1,000 lifetime prevalence reported by Griebling [5]. The difference in prevalence may be due to the differences in the populations studied (Dutch registry and US hospital and ambulatory care records vs. survey) and changes in treatment patterns such as an increased rate of outpatient care for UTIs (greater numbers seeking care) in the past decade [5]. In a prospective study of 179 women in Finland [19], 44\% of patients had recurrent UTIs compared with $23 \%$ of individuals with T2DM in SHIELD. The difference in recurrence rates may be due to the difference in definition of recurrence, as the Finland study defined recurrence as an episode of UTI at least 1 month after the index episode [19]. Other investigations have reported risk factors for recurrent vaginitis but found different predictors than the SHIELD study. A prospective study of 104 women with a history of UTIs found that bacteriuria, pyuria, and intercourse dramatically increased the likelihood of recurrent UTIs [15]. A study of 49 women with recurrent UTIs found that $76 \%$ had dysfunctional voiding [16], and a study of 1,140 women with pelvic floor dysfunction found that the pelvic dysfunction was associated with recurrent UTIs [20]. The SHIELD study found that self-reported overactive bladder/incontinence was a risk factor for recurrent UTIs, supporting an association 
Grandy et al. Journal of Diabetes Research \& Clinical Metabolism 2013,

with dysfunctional voiding. However, SHIELD did not collect data on sexual activity, menstruation habits, or pelvic dysfunction. The SHIELD study also provided evidence of risk factors that are readily known in routine clinical practice and can be used to assist in the identification of individuals (demographics and comorbid conditions) who are likely to have recurrent infections so that appropriate preventive care can be implemented.

The presence of COPD and revascularization (angioplasty, stents, bypass surgery) were significant predictors of recurrent UTIs compared with non-recurrent UTIs (83\% decreased risk of recurrent UTI for revascularization and 93\% decreased risk for COPD) for women. This association may be due to use of antibiotics since antibiotics are generally administered in the US for 5 to 10 days for acute exacerbations of COPD [21]; however, future research is needed to test this hypothesis. T2DM women who were treated with insulin plus oral anti-diabetic medications had significantly increased odds of recurrent vaginitis compared with both those with non-recurrent vaginitis and no vaginitis. This association may be due to poor glucose control or indicative of difficult-to-treat T2DM patients since they were receiving insulin. Further research is needed to evaluate the impact of glucose control on recurrence of vaginitis.

There are limitations to the study that should be considered. The diagnosis of diabetes, other comorbid conditions, and infections (UTIs and genital infections) were self-reported and could not be validated with medical record review. However, this bias is similar between the groups compared in this study. Also, previous studies have shown good correlation between self-reported health conditions and objective laboratory testing $[12,22,23]$. Household panels, like the TNS NFO panel, tend to under-represent the very wealthy and very poor segments of the population and do not include military or institutionalized individuals. Also, survey respondents are more likely to have healthier lifestyles than non-respondents [24] which may bias the generalizability of the study findings. However, the SHIELD population was weighted to represent the US census on age, gender, and household size, which may partially correct this bias. Information on sexual activity was not collected in the SHIELD survey so this factor could not be assessed as a potential predictor of urinary or genital infections. Even in this large cohort study, some of the $95 \% \mathrm{Cls}$ are wide, so robust estimates for the odds of infection or recurrence of infection are limited for certain characteristics. For example, there were a few women with recurrent or non-recurrent vaginitis who reported a stroke, resulting in a $95 \% \mathrm{Cl}$ interval that reached from 1 to 41.

In conclusion, UTIs and genital infections are prevalent among adults with T2DM, and recurrence of these infections is common in T2DM, especially among women. Predisposing characteristics of individuals with recurrent infections may be helpful in identifying those at risk before recurrence to reduce the burden on patients, physicians, and the healthcare system.

\section{List of Abbreviations}

BMI: Body mass index

COPD: Chronic obstructive pulmonary disease

DM: Diabetes mellitus

OR: Odds ratio

SHIELD: Study to Help Improve Early evaluation and

management of risk factors Leading to Diabetes

T2DM: Type 2 diabetes mellitus

UTI: Urinary tract infection

\section{Competing interests}

SG and EH are employees and stockholders of AstraZeneca LP. KMF received research funds from AstraZeneca LP to conduct the analysis.

\section{Authors' contributions}

SG made substantial contribution to the research design of the study, acquisition of the data, assisted in the data interpretation, reviewed and critically revised the manuscript, and gave final approval of the manuscript. KMF made substantial contributions to the research design, managed the data analysis, interpreted the data, drafted the manuscript, and revised and approved the final version of the manuscript. EH made substantial contributions to the study design, interpretation of the data, critically reviewed the manuscript, and approved the final manuscript.

\section{Acknowledgement}

This research was supported by AstraZeneca LP. Members of the SHIELD Study Group are: Harold Bays, MD, Louisville Metabolic and Atherosclerosis Research Center, Louisville, KY; Debbra D. Bazata, RD, CDE, St. Luke's Primary Care South, Overland Park, KS; James R. Gavin III, MD, PhD, Emory University School of Medicine, Atlanta, GA; Andrew J. Green, MD, Midwestern Endocrinology, Overland Park, KS; Sandra J. Lewis, MD, Northwest Cardiovascular Institute, Portland, OR; Michael L. Reed, PhD, Vedanta Research, Chapel Hill, NC; and Helena W. Rodbard, MD, Endocrine and Metabolic Associates, Rockville, MD. Tina Fanning of Vedanta Research, Chapel Hill, NC, also contributed to this report, performing data collection and analysis.

\section{Publication history}

Received: 18-Dec-2012 Revised: 23-Jan-2013

Accepted: 25-Jan-2013 Published: 29-Jan-2013

\section{References}

1. Platt $F W$ and Keating K N: Differences in physician and patient perceptions of uncomplicated UTI symptom severity: understanding the communication gap. Int J Clin Pract 2007, 61:303-8. | Article | PubMed

2. Foxman B: Epidemiology of urinary tract infections: incidence, morbidity, and economic costs. Am J Med 2002, 113 Suppl 1A:5S-13S. | Article | PubMed

3. Kunin C M: Urinary tract infections in females. Clin Infect Dis 1994, 18:1-10. | Article | PubMed

4. Engel J D and Schaeffer A J: Evaluation of and antimicrobial therapy for recurrent urinary tract infections in women. Urol Clin North Am 1998, 25:685-701, x. | Article | PubMed

5. Griebling T L: Urologic diseases in america project: trends in resource use for urinary tract infections in men. J Urol 2005, 173:1288- 


\section{4. | Article | PubMed}

6. Norinder B S, Norrby R, Palmgren A C, Hollenberg S, Eriksson U and Nord C E: Microflora changes with norfloxacin and pivmecillinam in women with recurrent urinary tract infection. Antimicrob Agents Chemother 2006, 50:1528-30. | Article | PubMed Abstract | PubMed Full Text

7. Uehara $S$, Monden $K$, Nomoto $K$, Seno $Y$, Kariyama $R$ and Kumon $H$ : A pilot study evaluating the safety and effectiveness of Lactobacillus vaginal suppositories in patients with recurrent urinary tract infection. Int J Antimicrob Agents 2006, 28 Suppl 1:S30-4. | Article | PubMed

8. Del-Cura Gonzalez I, Garcia-de-Blas Gonzalez F, Cuesta T S, Martin Fernandez J, Del-Alamo Rodriguez J M, Escriva Ferrairo R A, Del Canto De-Hoyos Alonso M, Arenas L B, Barrientos R R, Wiesmann E C, et al: Patient preferences and treatment safety for uncomplicated vulvovaginal candidiasis in primary health care. BMC Public Health 2011, 11:63. | Article | PubMed Abstract | PubMed Full Text

9. Eckert L O: Clinical practice. Acute vulvovaginitis. N Engl J Med 2006, 355:1244-52. | Article | PubMed

10. Stapleton A: Urinary tract infections in patients with diabetes. Am J Med 2002, 113 Suppl 1A:80S-84S. | Article | PubMed

11. Schneeberger C, Stolk R P, Devries J H, Schneeberger P M, Herings $\mathrm{R} \mathrm{M}$ and Geerlings $S \mathrm{E}$ : Differences in the pattern of antibiotic prescription profile and recurrence rate for possible urinary tract infections in women with and without diabetes. Diabetes Care 2008 31:1380-5. | Article | PubMed Abstract | PubMed Full Text

12. Bays $\mathrm{HE}$ E, Chapman $\mathrm{R} H$ and Grandy S: The relationship of body mass index to diabetes mellitus, hypertension and dyslipidaemia: comparison of data from two national surveys. Int J Clin Pract 2007, 61:737-47. | Article | PubMed Abstract | PubMed Full Text

13. Bays H E, Bazata D D, Clark N G, Gavin J R, 3rd, Green A J, Lewis S J, Reed M L, Stewart W, Chapman R H, Fox K M and Grandy S: Prevalence of self-reported diagnosis of diabetes mellitus and associated risk factors in a national survey in the US population: SHIELD (Study to Help Improve Early evaluation and management of risk factors Leading to Diabetes). BMC Public Health 2007, 7:277. | Article | PubMed Abstract | PubMed Full Text

14. US Census Bureau: Annual Supplement to the Current Population Survey: Census Bureau Resident Population Estimates of the United States. Washington, DC: US Census Bureau 2003.

15. Czaja C A, Stamm W E, Stapleton A E, Roberts P L, Hawn T R, Scholes D, Samadpour M, Hultgren S J and Hooton T M: Prospective cohort study of microbial and inflammatory events immediately preceding Escherichia coli recurrent urinary tract infection in women. J Infect Dis 2009, 200:528-36. | Article | PubMed

16. Akkad T, Pelzer A E, Mitterberger M, Rehder P, Leonhartsberger $N$, Bartsch $\mathrm{G}$ and Strasser $\mathrm{H}$ : Influence of intravesical potassium on pelvic floor activity in women with recurrent urinary tract infections: comparative urodynamics might lead to enhanced detection of dysfunctional voiding. BJU Int 2007, 100:1071-4. | Article | PubMed

17. Alexiou Z, Mouktaroudi M, Koratzanis G, Papadopoulos A, Kavatha D, Kanellakopoulou K, Giamarellou H and Giamarellos-Bourboulis E $\mathrm{J}$ : The significance of compliance for the success of antimicrobial prophylaxis in recurrent lower urinary tract infections: the Greek experience. Int J Antimicrob Agents 2007, 30:40-3. | Article | PubMed

18. Mohsin $\mathrm{R}$ and Siddiqui $\mathrm{K} M$ : Recurrent urinary tract infections in females. J Pak Med Assoc 2010, 60:55-9. | Article | PubMed

19. Ikaheimo R, Siitonen $A$, Heiskanen $T$, Karkkainen $U$, Kuosmanen $P$, Lipponen $\mathrm{P}$ and Makela $\mathrm{P} H$ : Recurrence of urinary tract infection in a primary care setting: analysis of a 1-year follow-up of 179 women. Clin Infect Dis 1996, 22:91-9. | Article | PubMed

20. Haylen B T, Lee J, Husselbee S, Law M and Zhou J: Recurrent urinary tract infections in women with symptoms of pelvic floor dysfunction. Int Urogynecol J Pelvic Floor Dysfunct 2009, 20:837-42. | Article I PubMed

21. Wenzel R P, Fowler A A, 3rd and Edmond M B: Antibiotic prevention of acute exacerbations of COPD. N Engl J Med 2012, 367:340-7. | Article I PubMed
22. Bays H E, Chapman R H, Fox K M and Grandy S: Comparison of self-reported survey (SHIELD) versus NHANES data in estimating prevalence of dyslipidemia. Curr Med Res Opin 2008, 24:1179-86. I Article I PubMed

23. Falk D E, Yi H Y and Hiller-Sturmhofel S: An epidemiologic analysis of co-occurring alcohol and tobacco use and disorders: findings from the National Epidemiologic Survey on Alcohol and Related Conditions. Alcohol Res Health 2006, 29:162-71. | PDF | PubMed

24. Tolonen H, Dobson A and Kulathinal S: Effect on trend estimates of the difference between survey respondents and non-respondents: results from 27 populations in the WHO MONICA Project. Eur J Epidemiol 2005, 20:887-98. | Article | PubMed

\section{Citation:}

Grandy S, Fox K M and Hardy E: Prevalence and recurrence of urinary tract and genital infections among adults with and without type 2 diabetes mellitus in the general population: a longitudinal cohort study. Journal of Diabetes Research and Clinical Metabolism 2013, 2:5.

http://dx.doi.org/10.7243/2050-0866-2-5 